\title{
Upregulation of AQP2 mediated by transcription factor FOXO1 inhibits TGF- $\beta$-induced fibrosis in human urothelial cells
}

\author{
YUE QIU, ZHAOQUAN XING* ${ }^{*}$ ZHAOXIN GUO* and ZHAOXU LIU \\ Department of Urology, Qilu Hospital of Shandong University, Jinan, Shandong 250012, P.R. China
}

Received March 4, 2021; Accepted June 11, 2021

DOI: $10.3892 / \mathrm{etm} .2021 .10824$

\begin{abstract}
Bladder outlet obstruction (BOO) is a common urological disease, and inhibition of TGF- $\beta$-induced bladder tissue fibrosis may serve as an alternative strategy for BOO treatment. Aquaporin (AQP)2 was reported to be aberrantly expressed in rat $\mathrm{BOO}$, but its specific role was not clarified. The aim of the present study was to explore the role of AQP2 in TGF- $\beta$-induced urothelial cell fibrosis and elucidate the potential underlying mechanism. The SV-HUC-1 human urinary tract epithelial cell line was treated with TGF- $\beta 1$ to establish an in vitro model of bladder fibrosis. Cell Counting Kit- 8 and wound healing assays were performed to measure cell viability and migration, respectively. Cell transfection was conducted to silence/overexpress AQP2 and Forkhead box O1 (FOXO1). Protein expression was measured using western blotting. Luciferase reporter and chromatin immunoprecipitation assays were used to verify the predicted interaction between AQP2 and FOXO1. The present study found that AQP2 expression was downregulated in TGF- $\beta 1$-treated urothelial cells. Overexpression of AQP2 significantly suppressed cell viability, migration and epithelial-to-mesenchymal transition in TGF- $\beta 1$-treated SV-HUC-1 cells. In addition, FOXO1 overexpression exerted similar effects as AQP2 overexpression on TGF- $\beta$-treated SV-HUC-1 cells, but these changes were partially abolished by AQP2 knockdown. It was also found that FOXO1 was able to bind to the AQP2 promoter and regulate AQP2 expression. In conclusion, the transcription factor FOXO1 may upregulate AQP2 expression, thereby inhibiting TGF- $\beta$-induced fibrosis in human urothelial cells. The findings of the present study may provide a novel potential strategy for the clinical treatment of BOO by targeting AQP2.
\end{abstract}

Correspondence to: Dr Zhaoxu Liu, Department of Urology, Qilu Hospital of Shandong University, 107 Cultural West Road, Jinan, Shandong 250012, P.R. China

E-mail: liuzhaoxu2021@163.com

*Contributed equally

Key words: bladder fibrosis, chitinase 3 like 1, forkhead box protein $\mathrm{O} 1$, transforming growth factor- $\beta 1$, epithelial-tomesenchymal transition

\section{Introduction}

Bladder outlet obstruction (BOO) is a common urological disease that is mainly caused by benign prostate hyperplasia, contracture of the bladder neck, stricture of the urethra, deformity of the lower urinary tract and compression by organs around the bladder (1). Long-term BOO may result in bladder structure remodeling and bladder detrusor muscle dysfunction, which may in turn lead to a series of urinary tract symptoms, including frequent urination, urination urgency and urinary incontinence, severely compromising the quality of life of the patients (2). Currently, BOO affects $\sim 1.1$ billion individuals worldwide, with the occurrence of $\mathrm{BOO}$ and associated lower urinary tract symptoms increasing annually $(3,4)$. During the pathological process of BOO, smooth muscle hypertrophy occurs in an attempt to overcome the increased urethral resistance, which leads to the eventual decompensation to fibrosis (5). Bladder tissue fibrosis is a pathological consequence that occurs in most, if not all, cases of BOO. It has been previously reported that TGF- $\beta 1$-induced porcine bladder urothelial cells successfully simulated the process of bladder fibrosis, emphasizing the critical role of TGF- $\beta 1$ in the induction of fibrosis (6). Further reports revealed that TGF- $\beta 1$ serves an important role in the development of bladder fibrosis, and inhibition of TGF- $\beta 1$-induced bladder tissue fibrosis may prove to be a viable strategy for $\mathrm{BOO}$ treatment $(7,8)$.

Aquaporin (AQP)2 belongs to the AQP family of proteins, which includes 13 subtypes (AQP0-12). Serving as classical water channel proteins that are ubiquitously expressed in mammalian tissues, AQPs act as hydrophobic and integral membrane channel proteins that are involved in fluid transport $(9,10)$. AQP2 has been found to be expressed in various tissues, including fallopian tubes, pancreatic islets, small intestine, kidney and bladder (10). Kim et al (11) previously investigated the regulatory mechanism of AQPs in a $\mathrm{BOO}$ rat model, and found that the expression of both AQP2 and AQP3 were elevated in the rat urinary bladder, indicating that AQP2/3 may be involved in BOO. In addition, AQP2 was mainly expressed in the epithelial cell layer and detrusor smooth muscle (11). However, the effects of AQP2 on the physiology of bladder dysfunction remain unclear and require further investigation.

hTFtarget analysis (http://bioinfo.life.hust.edu. $\mathrm{cn} / \mathrm{hTFtarget} \# ! /$ ) identified a predicted interaction between the Forkhead box O1 (FOXO1) and the AQP2 promoter. FOXO1 
is a member of the FOXO protein family that is known to be involved in various cellular processes, including cell proliferation and differentiation (12). The FOXO1 gene is located on chromosome 13 and translates into FOXO1 protein. Substantial evidence indicates that the function of FOXO1 depends on the interaction with DNA, thereby modulating downstream targets (13). It has been reported that FOXO1 may serve as a tumor suppressor in urothelial cells to prevent urothelial carcinogenesis and cancer growth (14). Additionally, FOXO1 was found to be closely involved in fibrosis of multiple organs and has been considered to be a promising target for anti-fibrosis therapy (15). However, at present, the role of FOXO1 in bladder fibrosis remains unclear, and the potential effects of FOXO1 on $\mathrm{BOO}$ warrant further investigation.

On the basis of the aforementioned findings, the present study hypothesized that AQP2 may combine with FOXO1, which may in turn be involved in the pathophysiology of bladder dysfunction as a result of BOO. The aim of the present study was to explore the role of AQP2 in TGF- $\beta$-induced urothelial cell fibrosis and its potential mechanism of action.

\section{Materials and methods}

Cell culture and treatment. The SV-HUC-1 human urinary tract epithelial cell line was obtained from American Type Culture Collection and cultured in Ham's F12 medium (Sigma-Aldrich; Merck KGaA) supplemented with 7\% fetal bovine serum (HyClone; Cytiva) and 1\% penicillin/streptomycin. SV-HUC-1 cells were cultured in a humidified incubator with $5 \% \mathrm{CO}_{2}$ at $37^{\circ} \mathrm{C}$. SV-HUC-1 cells were then stimulated with TGF- $\beta 1$ (R\&D Systems, Inc.) at 1,5 and $10 \mathrm{ng} / \mathrm{ml}$ to mimic bladder fibrosis.

Reverse transcription-quantitative PCR (RT-PCR) analysis. Total RNA was extracted from SV-HUC-1 cells using TRIzol ${ }^{\circledR}$ reagent (Invitrogen; Thermo Fisher Scientific, Inc.). In total, $1 \mu \mathrm{g}$ RNA was reverse-transcribed into cDNA in accordance with the protocols of PrimeScript ${ }^{\mathrm{TM}}$ RT reagent kit (Takara Bio, Inc.). Subsequently, qPCR was performed using SYBR ${ }^{\circledR}$ Premix EX Taq ${ }^{\mathrm{TM}}$ II (Takara Bio, Inc.). The primers used were as follows: AQP2, forward, 5'-TGGGCCATATGTGCT ATGGAGA-3' and reverse, 5'-AAGGACACTCAGGTG CCAGGA-3'; FOXO1, forward, 5'-GGGTTAGTGAGCAGG TTACAC-3' and reverse, 5'-TCCAATGGCACAGTCCTT ATC-3'; and $\beta$-actin, forward, 5'-CATCCACGAAACTAC CTTCAACTCC-3' and reverse, 5'-GAGCCGCCGATCCAC ACG-3'. For PCR, the following thermocycling program was adopted: Initial denaturation at $94^{\circ} \mathrm{C}$ for $5 \mathrm{~min} ; 30$ cycles of denaturation at $94^{\circ} \mathrm{C}$ for $30 \mathrm{sec}$, annealing at $65^{\circ} \mathrm{C}$ for $30 \mathrm{sec}$ and extension at $72^{\circ} \mathrm{C}$ for $30 \mathrm{sec}$ and a final extension step at $72^{\circ} \mathrm{C}$ for $10 \mathrm{~min}$. The relative mRNA expression of AQP2 and FOXO1 was calculated using the $2^{-\Delta \Delta \mathrm{Cq}}$ method (16), followed by normalization using $\beta$-actin as the reference gene.

Western blotting. Total protein was extracted from SV-HUC-1 cells using RIPA lysis buffer (Nanjing KeyGen Biotech Co., Ltd.). After determining the protein concentration using a bicinchoninic acid protein assay kit (Nanjing KeyGen Biotech Co., Ltd.), equal amounts of protein $(30 \mu \mathrm{g} /$ lane $)$ were separated by $12 \%$ SDS-PAGE and transferred onto PVDF membranes.
The membranes were blocked with 5\% skimmed milk at room temperature for $2 \mathrm{~h}$, followed by incubation with primary antibodies against AQP2 (1:500; cat. no. ab199975; Abcam), E-cadherin (1:10,000; cat. no. ab40772; Abcam), N-cadherin (1:1,000; cat. no. ab18203; Abcam), cytokeratin 5 (1:10,000; cat. no. ab52635; Abcam), fibronectin (1:1,000; cat. no. ab2413; Abcam), $\alpha$-SMA (1:10,00; cat. no. ab5831; Abcam), FOXO1 (1:1,000; cat. no. ab39670; Abcam) and GAPDH (1:1,000; cat. no. ab8245; Abcam), at $4^{\circ} \mathrm{C}$ overnight. On the following day, the membranes were washed with TBS with $0.1 \%$ Tween-20 (TBST) for $5 \mathrm{~min}$ three times and incubated with horseradish peroxidase-conjugated goat anti-rabbit secondary antibodies (1:20,000; cat. no. ZB-2301; OriGene Technologies, Inc.) at room temperature for $2 \mathrm{~h}$. The blots were visualized using the ECL chemiluminescent substrate (GE Healthcare).

Cell transfection. To overexpress or inhibit the expression of AQP2, pcDNA 3.1-AQP2 and short hairpin (sh) RNA targeting AQP2 (shRNA-AQP2; cat. no. C01001) were obtained from Shanghai GenePharma Co., Ltd. When the cell confluence reached $60-70 \%$, cells were transfected with pcDNA $3.1-A Q P 2$ (15 nM), shRNA-AQP2 (500 ng/ $\mu \mathrm{l})$ or their corresponding negative controls (NC; pcDNA-NC (15 nM) and shRNA-NC (500 ng/ $\mu \mathrm{l}$ ) using Lipofectamine ${ }^{\circledR} 3000$ (Invitrogen; Thermo Fisher Scientific, Inc.) according to the manufacturer's instructions in a humidified incubator with $5 \% \mathrm{CO}_{2}$ at $37^{\circ} \mathrm{C}$ for $48 \mathrm{~h}$. Western blot analysis was performed $48 \mathrm{~h}$ after transfection to examine transfection efficacy.

Cell viability assay. Cell viability was examined using the Cell Counting Kit-8 (CCK-8) assay (Dojindo Molecular Technologies, Inc.) according to the manufacturer's instructions. In brief, the transfected cells were incubated with $5 \mathrm{ng} / \mathrm{ml} \mathrm{TGF}-\beta 1$ for 12,24 and $36 \mathrm{~h}$ at $37^{\circ} \mathrm{C}$. A total of $10 \mu \mathrm{l}$ CCK- 8 solution were then added to each well and the cells were incubated for another $3 \mathrm{~h}$. The absorbance of each well was then detected at $450 \mathrm{~nm}$ using a microplate reader (Bio-Rad Laboratories, Inc.).

Cell migration assay. Cell migration ability was measured using the wound healing assay. Briefly, the transfected cells were inoculated into six-well plates $\left(1 \times 10^{6}\right.$ cells/well $)$ for incubation. When the cell confluence reached $100 \%$, a linear scratch was created using a $200-\mu 1$ pipette tip. The cells were washed with PBS before being incubated in FBS-free medium for $24 \mathrm{~h}$. Images were captured at 0 and $24 \mathrm{~h}$ using a light microscope (magnification, x100).

Chromatin immunoprecipitation (ChIP) assay. ChIP assay was performed using the SimpleChIP ${ }^{\circledR}$ Plus Sonication Chromatin IP kit (Cell Signaling Technology, Inc.) according to the manufacturer's instructions. After the indicated treatment, SV-HUC-1 cells were fixed with $4 \%$ formaldehyde at room temperature for $10 \mathrm{~min}$ and sonicated using a $10 \mathrm{sec}$ on and $10 \mathrm{sec}$ off mode for 12 cycles on ice. An antibody against FOXO1 (cat. no. ab39670; Abcam) was used for the immunoprecipitation experiments before the extent of AQP2 enrichment was analyzed by RT-qPCR. IgG (cat. no. ab90285; Abcam) was used as an isotype control and input DNA was amplified for each sample in parallel runs. 
A

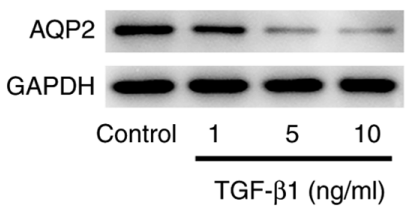

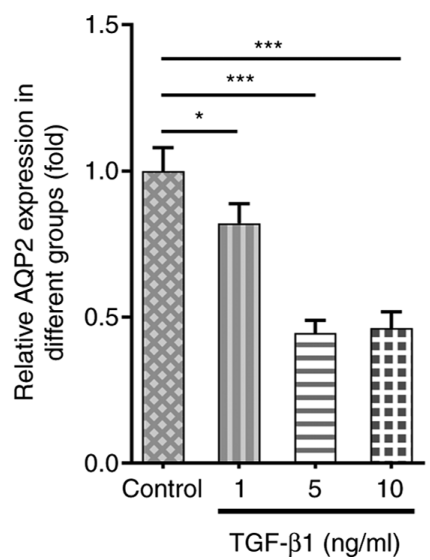

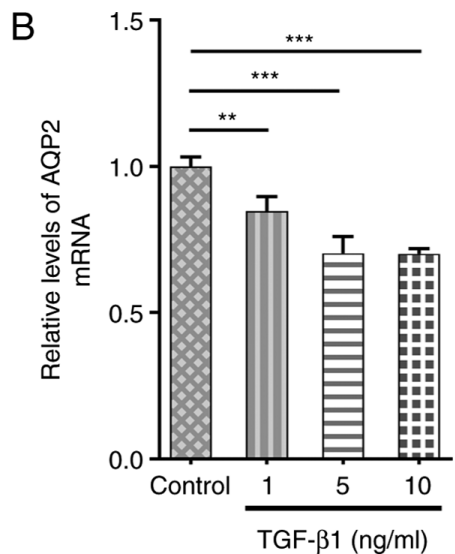

Figure 1. AQP2 expression is downregulated by TGF- $\beta 1$ treatment in SV-HUC-1 cells. The SV-HUC-1 human urinary tract epithelial cell line was treated with TGF- $\beta 1$ at $0,1,5$ and $10 \mathrm{ng} / \mathrm{ml}$, and the protein and mRNA expression levels of AQP2 were measured using (A) western blotting and (B) reverse transcription-quantitative PCR, respectively. ${ }^{*} \mathrm{P}<0.05,{ }^{* *} \mathrm{P}<0.01$ and ${ }^{* * *} \mathrm{P}<0.001$ vs. control. AQP2, aquaporin 2.

Luciferase reporter assay. A predicted interaction between FOXO1 and AQP2 promoter was found using the hTFtarget online tool (http://bioinfo.life.hust.edu.cn/hTFtarget\#!/). The pGL3 plasmid (Promega Corporation) containing the AQP2 promoter region element was generated by site-directed mutagenesis and subcloned into the pGL3-basic luciferase reporter vector. A mutant type (MUT) and wild-type (WT) AQP2 promoter vector were produced by GeneCopoeia Co., Ltd. (Guangzhou, China). These plasmids (500 ng/ $\mu \mathrm{l}$ ) were transfected into SV-HUC-1 cells alongside pcDNA3.1-FOXO1 (15 nM) using Lipofectamine ${ }^{\circledR} 3000$ (Invitrogen; Thermo Fisher Scientific, Inc.) according to the manufacturer's instructions. The luciferase reporter activity was detected using the Dual-luciferase reporter assay (Promega Corporation) according to the manufacturer's instructions. The luciferase intensities were determined by normalizing the Firefly luminescence to Renilla luminescence.

Statistical analysis. Data are presented as the mean \pm SD from at least three independent experiments. Statistical analysis was performed using SPSS software, version 20.0 (IBM Corp.). Comparisons among different groups were analyzed by unpaired Student's t-test or one-way ANOVA followed by Tukey's post hoc test. $\mathrm{P}<0.05$ was considered to indicate a statistically significant difference.

\section{Results}

AQP2 expression is downregulated in TGF- $\beta 1$-treated $S V-H U C-1$ cells. To establish the bladder fibrosis cell model in vitro, TGF- $\beta 1$ was applied to stimulate SV-HUC-1 human urinary tract epithelial cells. As shown in Fig. 1A and B, AQP2 expression was downregulated at both the protein and mRNA levels following TGF- $\beta 1$ stimulation compared with untreated cells. Since there was no significant difference in AQP2 expression between TGF- $\beta 1$ treatment at 5 and $10 \mathrm{ng} / \mathrm{ml}, 5 \mathrm{ng} / \mathrm{ml}$ TGF- $\beta 1$ treatment was selected for further experiments.

AQP2 overexpression reduces cell viability, inhibits migration and epithelial-to-mesenchymal transition (EMT) in TGF- $\beta 1$-induced SV-HUC-1 cells. To explore the role of AQP2 in bladder fibrosis, SV-HUC-1 cells were transfected with pcDNA3.1-AQP2, which significantly increased the protein expression of AQP2 (Fig. 2A). The cells were then treated with TGF- $\beta 1$, with or without AQP2 overexpression. TGF- $\beta 1$ treatment markedly increased cell viability, which was partially prevented by AQP2 overexpression (Fig. 2B). The wound healing assay revealed that TGF- $\beta 1$ notably promoted cell migration ability, which was also partially blocked by AQP2 overexpression (Fig. 2C and D). Western blot analysis revealed reduced expression of E-cadherin and increased expression of $\mathrm{N}$-cadherin after TGF- $\beta 1$ treatment, suggesting that TGF- $\beta 1$ promoted EMT in SV-HUC-1 cells. Consistently, AQP2 overexpression also partly prevented the effects of TGF- $\beta 1$ on E-cadherin and N-cadherin expression (Fig. 2E). TGF- $\beta 1$ treatment was also found to induce bladder fibrosis, as the protein expression level of cytokeratin was decreased, whereas the expression of fibronectin (FN) and $\alpha$-smooth muscle actin $(\alpha$-SMA) protein levels was increased following TGF- $\beta 1$ treatment (Fig. 2F). These aforementioned changes were also reversed by AQP2 overexpression.

FOXO1 binds to the AQP2 promoter to regulate AQP2 expression. Using the hTFtarget online tool (http://bioinfo. life.hust.edu.cn/hTFtarget\#!/), a predicted interaction between FOXO1 and AQP2 promoter was found (Fig. 3A), which was verified using luciferase reporter and ChIP assays (Fig. 3B and C). In addition, SV-HUC-1 cells were transfected with pcDNA3.1-FOXO1 to overexpress FOXO1 (Fig. 3D). FOXO1 overexpression was found to significantly increase both the protein and mRNA expression levels of AQP2 in TGF- 31 -induced SV-HUC-1 cells (Fig. 3E and F).

Inhibitory effects of FOXO1 overexpression on the viability, migration and fibrosis of TGF- $\beta 1$-induced $S V-H U C-1$ cells are partly abolished by AQP2 knockdown. In accordance with the interaction between FOXO1 and AQP2, the expression level of FOXO1 in this in vitro model of bladder fibrosis was also detected. The protein expression of FOXO1 was found to be significantly decreased following TGF- $\beta 1$ stimulation in SV-HUC-1 cells (Fig. 4A). shRNA-NC and shRNA-AQP-1/2 were subsequently used to transfect SV-HUC-1 cells. AQP2 


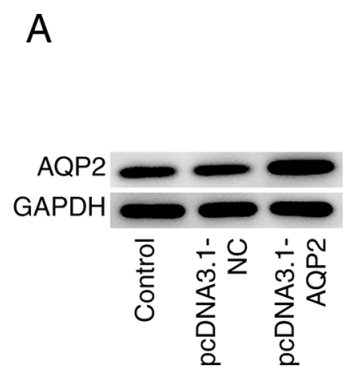

C
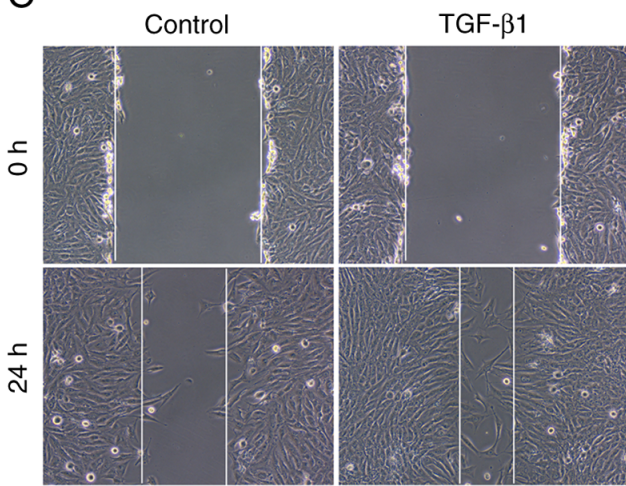

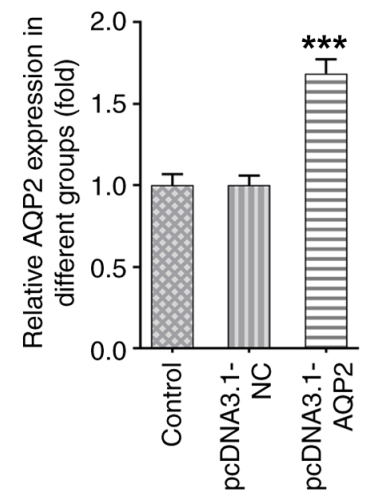

TGF- $\beta 1+p c D N A 3.1-N C$ TGF- $\beta 1+p c D N A 3.1-A Q P 2$
B $\rightarrow$ Control $\rightarrow$ TGF- $\beta 1+$ pCDNA3.1-NC

- TGF- $\beta 1$ - TGF- $\beta 1+$ pcDNA3.1-AQP2

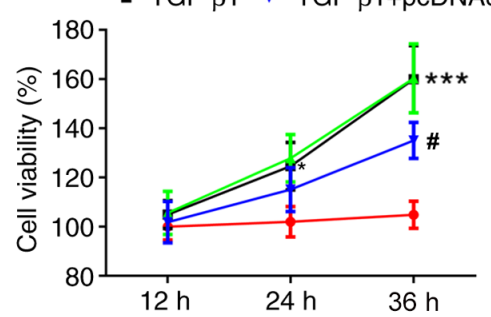

E

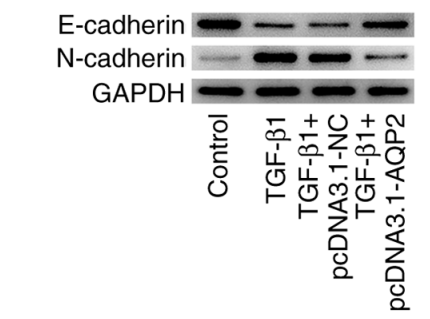

F

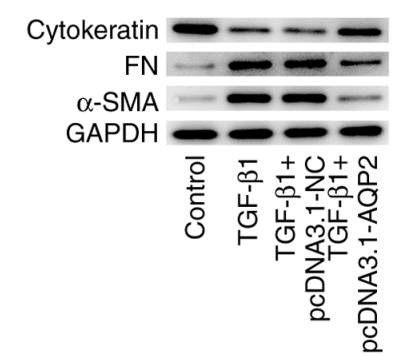

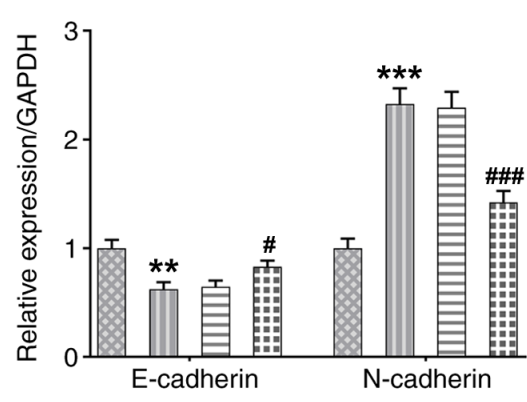

Control

m TGF- $\beta 1$

$\sqsupseteq$ TGF- $\beta 1+$ pcDNA3.1-NC

世

$\mathrm{D}$
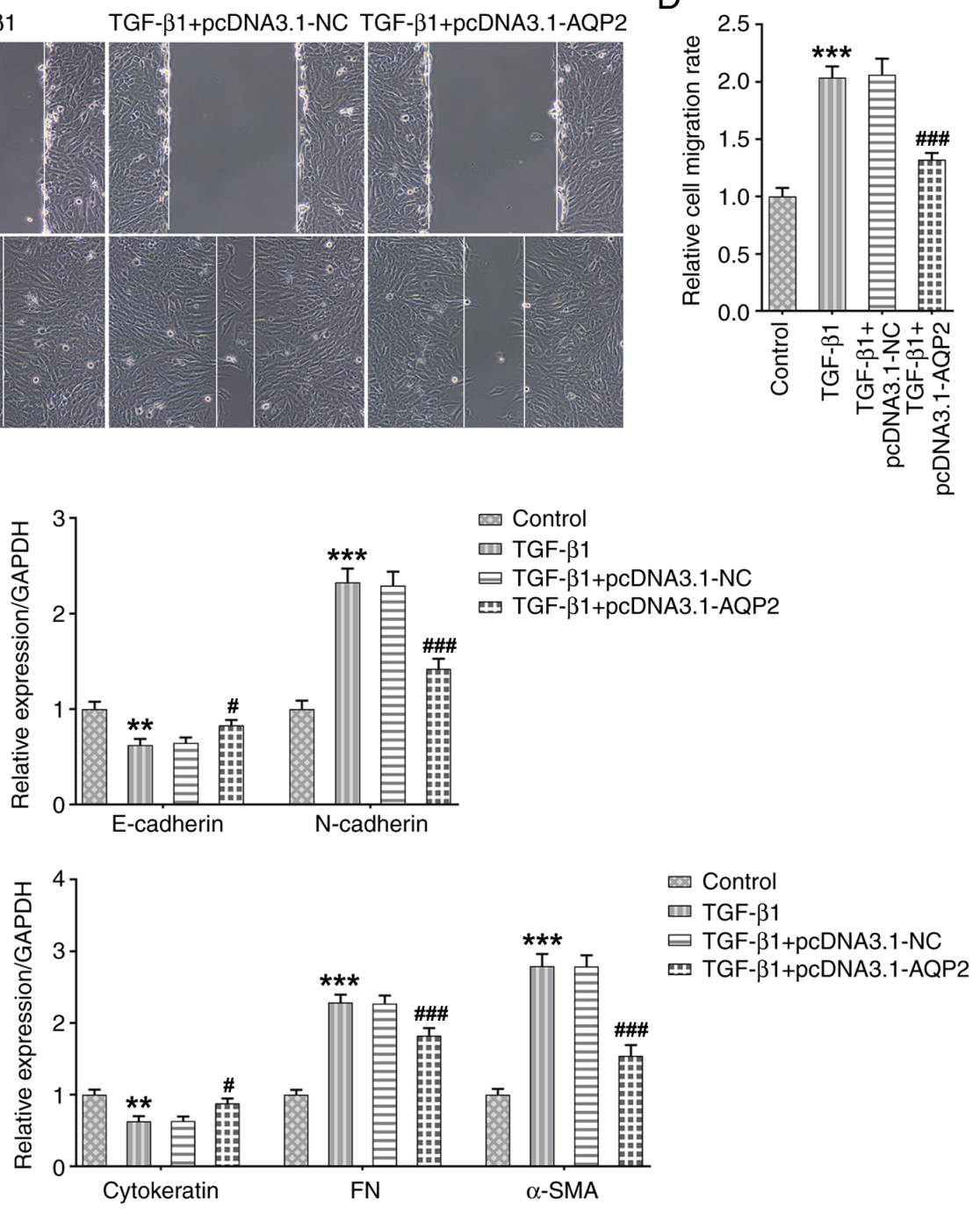

Figure 2. AQP2 overexpression reduces the viability and inhibits migration and epithelial-to-mesenchymal transition in TGF- $\beta 1$-induced SV-HUC- 1 cells. (A) SV-HUC-1 cells were transfected with pcDNA3.1-NC or pcDNA3.1-AQP2, and the protein expression of AQP2 was detected using western blotting. ${ }^{* * *} \mathrm{P}<0.001$ vs. pcDNA3.1-NC. (B-F) SV-HUC-1 cells were transfected with pcDNA3.1-NC or pcDNA3.1-AQP2 and treated with TGF- $\beta 1$. (B) Subsequently, cell viability was measured using Cell Counting Kit-8 assay. (C and D) Cell migration was measured using wound healing assay. (E and F) E-cadherin, N-cadherin, cytokeratin, FN and $\alpha$-SMA protein expression levels were detected and analyzed using western blotting. ${ }^{*} \mathrm{P}<0.05,{ }^{* * *} \mathrm{P}<0.01$ and ${ }^{* * * *} \mathrm{P}<0.001$ vs. control; ${ }^{\#} \mathrm{P}<0.05$ and ${ }^{\# \# \#} \mathrm{P}<0.001$ vs. TGF-ß1+pcDNA3.1-NC. AQP2, aquaporin 2; FN, fibronectin; AQP2, aquaporin 2; $\alpha$-SMA, $\alpha$-smooth muscle actin; NC, negative control.

protein expression was found to be significantly decreased in cells in the shRNA-AQP2-1 and shRNA-AQP2-2 groups (Fig. 4B). Due to its higher transfection efficacy, shRNA-AQP2-2 was selected for further experiments. SV-HUC-1 cells were then transfected with pcDNA-FOXO1, with or without shRNA-NC/shRNA-AQP2 co-transfection. As shown in Fig. 4C-E, FOXO1 overexpression significantly reversed the increased cell viability and migration abilities mediated by TGF- $\beta 1$ in SV-HUC-1 cells. In addition, FOXO1 overexpression increased the protein expression of E-cadherin 

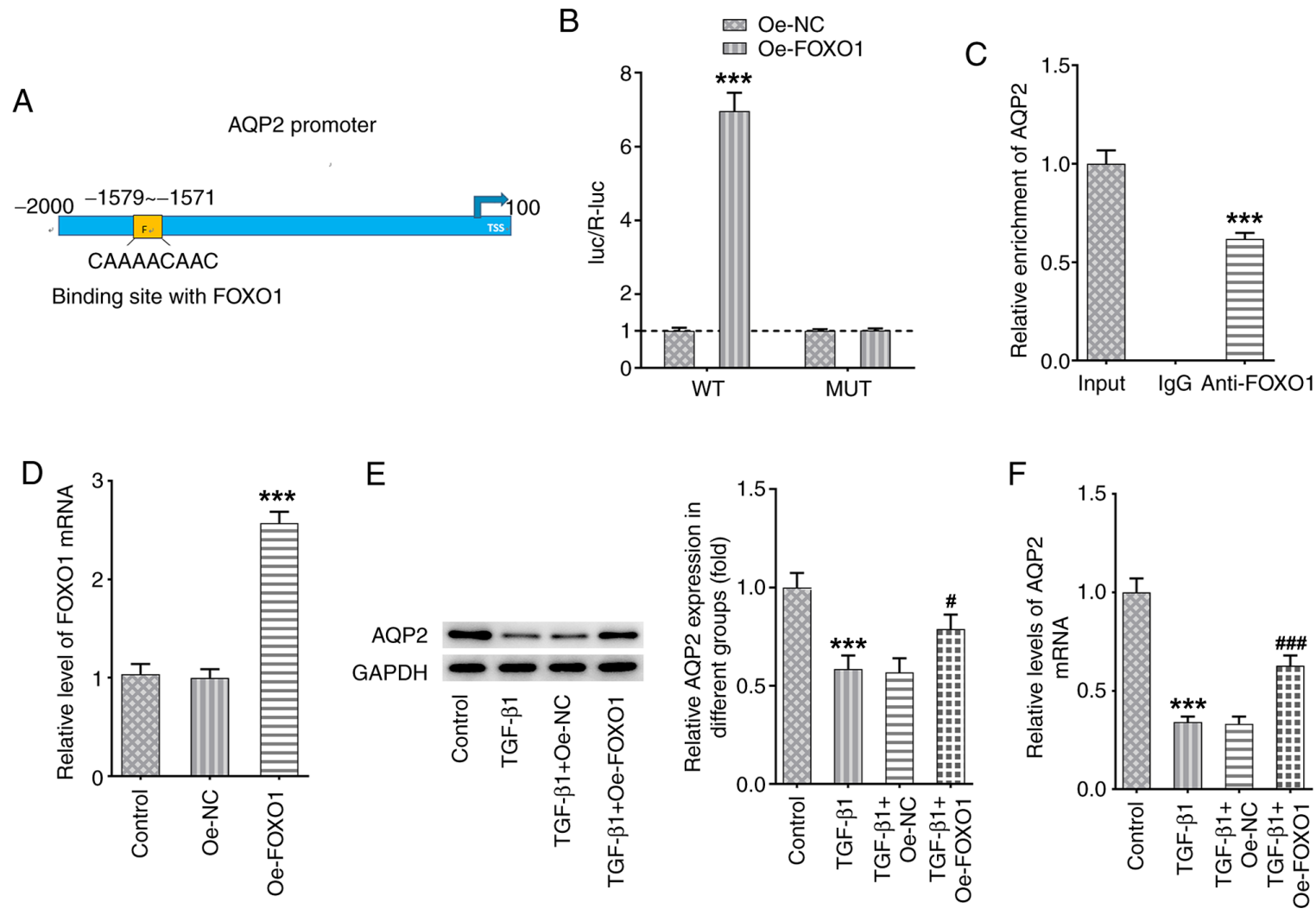

Figure 3. FOXO1 binds to the AQP2 promoter and regulates AQP2 expression. (A) hTFtarget online tool (http://bioinfo.life.hust.edu.cn/hTFtarget\#!/) predicted a potential interaction between FOXO1 and the AQP2 promoter. (B) Luciferase reporter assay was performed to verify this interaction. ${ }^{* * * *} \mathrm{P}<0.001 \mathrm{vs} . \mathrm{Oe}-\mathrm{NC}$. (C) Chromatin immunoprecipitation assay was performed to verify this interaction. ${ }^{* * * *} \mathrm{P}<0.001 \mathrm{vs.} \mathrm{IgG.} \mathrm{(D)} \mathrm{SV-HUC-1} \mathrm{cells} \mathrm{were} \mathrm{transfected} \mathrm{with} \mathrm{pcDNA3.1-NC}$ or pcDNA3.1-FOXO1, and the mRNA expression level of FOXO1 was measured using reverse transcription-quantitative PCR. ${ }^{* * *} \mathrm{P}<0.001$ vs. Oe-NC. SV-HUC-1 cells were treated with TGF- $\beta 1$ and transfected with pcDNA3.1-FOXO1, and then the (E) protein and (F) mRNA expression levels of AQP2 were measured using western blotting and reverse transcription-quantitative PCR, respectively. ${ }^{* * * *} \mathrm{P}<0.001$ vs. control; ${ }^{\#} \mathrm{P}<0.05$ and ${ }^{\# \# \#} \mathrm{P}<0.001$ vs. TGF- $\beta 1+\mathrm{Oe}-\mathrm{NC}$. FOXO1, Forkhead box protein O1; AQP2, aquaporin 2; NC, negative control; WT, wild-type; MUT, mutant.

and cytokeratin, whilst reducing the protein expression of $\mathrm{N}$-cadherin, FN and $\alpha$-SMA in TGF- $\beta 1$-induced SV-HUC-1 cells (Fig. 4F and G), suggesting that FOXO1 overexpression also suppressed EMT and bladder fibrosis. However, the suppressive effects of FOXO1 overexpression on cell viability, migration, EMT and fibrosis were abolished by AQP2 knockdown (Fig. 4C-G).

\section{Discussion}

The aim of the present study was to elucidate the role of AQP2 in TGF- $\beta 1$-treated SV-HUC- 1 cells and to investigate the potential mechanism underlying the association between AQP2 and bladder fibrosis. This was achieved by establishing an in vitro cell model of bladder fibrosis using the SV-HUC-1 cell line. A series of cellular function assays were performed, and the data revealed that $\mathrm{AQP} 2$ expression was downregulated following TGF- $\beta 1$-induced fibrosis, whereas AQP2 overexpression could alleviate the TGF- $\beta 1$-mediated effects on cell viability, migration and EMT. In addition, it was subsequently found that FOXO1 could directly bind to the AQP2 promoter to regulate AQP2 expression, where AQP2 upregulation downstream of FOXO1 inhibited the process of bladder fibrosis.

EMT is a process during which differentiated epithelial cells lose their epithelial phenotype and obtain mesenchymal cell-like characteristics (11). Additionally, EMT has also been previously associated with fibrosis (6). Mechanistically, loss of E-cadherin expression is the initial and pivotal step in EMT $(12,13)$. However, the expression of other epithelial markers, such as cytokeratin, is also lost $(12,13)$. This is accompanied by increases in the expression of mesenchymal markers, including $\mathrm{N}$-cadherin, FN and $\alpha$-SMA $(17,18)$. Recently, TGF- $\beta 1$ has been recognized as an inducer of EMT during fibrotic events in several organs, including the lung, kidney and liver (19-22). However, corresponding data regarding bladder fibrosis remain insufficient. To the best of our knowledge, only three previous studies have reported that TGF- $\beta 1$ may contribute to bladder fibrosis by mediating EMT $(6,18,23)$. In the present study, TGF- $\beta 1$ treatment resulted in the downregulation of E-cadherin and cytokeratin expression, which was accompanied by the upregulation of $\mathrm{N}$-cadherin, $\mathrm{FN}$ and $\alpha$-SMA expression, revealing that TGF- $\beta 1$ contributed to EMT in urinary tract epithelial cells, consistent with the aforementioned previous reports.

Binding of transcription factors to cognate DNA sequences in promoter and enhancer regions of targeted genes is a critical determinant of gene expression levels. FOXO1 is one of the classical transcription factors that are regulated by cell surface receptors. Promoter regions usually contain multiple transcription factor-binding sites (24). A present, only a small number of reports have revealed that FOXO1 can bind to the promoters of several genes, including STAT1 and cyclin D1, 
A
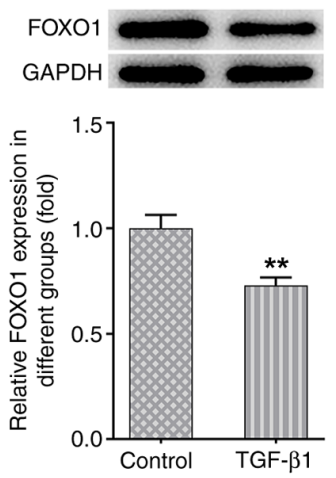

B

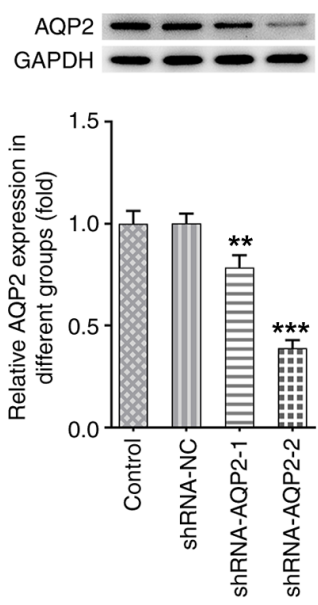

C

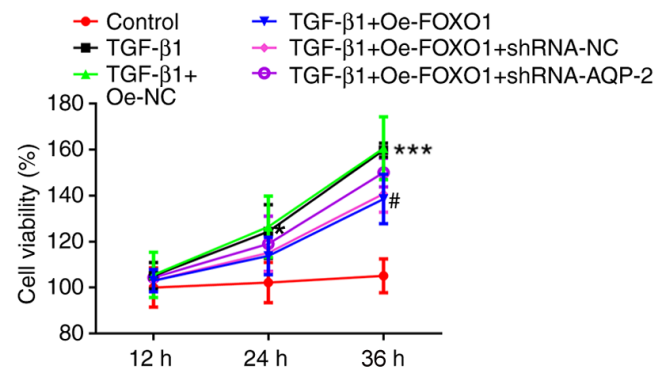

D
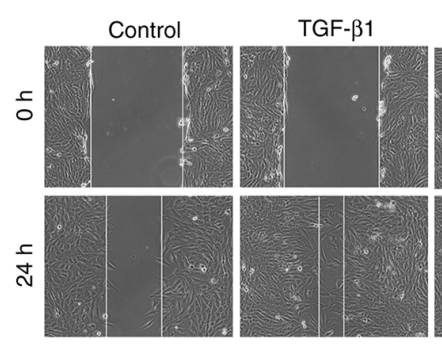

$\mathrm{F}$

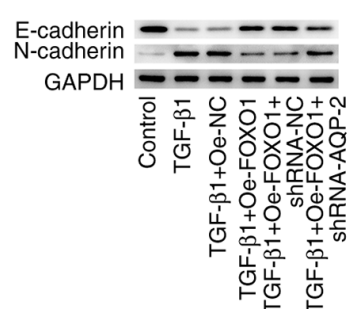

G

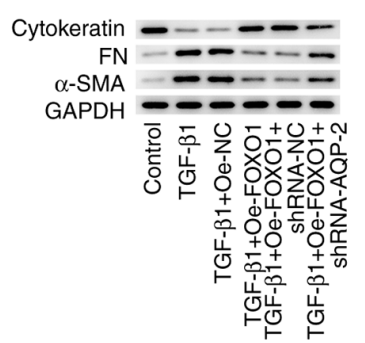

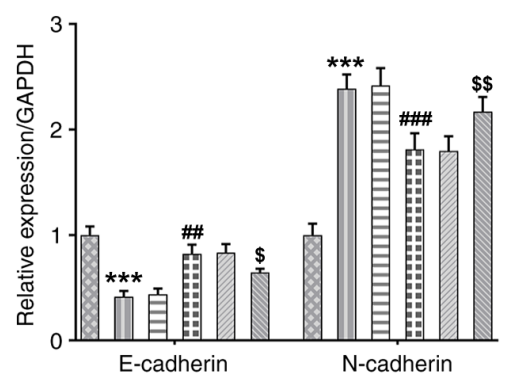

Control TGF- $\beta 1$

$\$ \$ \boxminus$ TGF- $\beta 1+\mathrm{Oe}-\mathrm{NC}$

世 TGF- $\beta 1+O e-F O X O 1$

TGF- $31+\mathrm{O} e-\mathrm{FOXO1+shRNA-NC}$

TGF-B1+Oe-FOXO1+shRNA-AQP-2
E

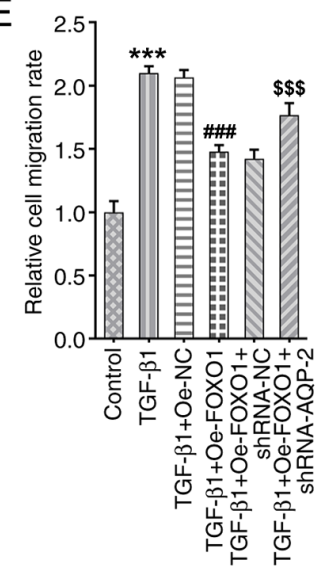

Figure 4. Inhibitory effects of FOXO1 overexpression on viability, migration and fibrosis in TGF- $\beta 1$-induced SV-HUC-1 cells are partly abolished by AQP2 knockdown. (A) FOXO1 protein expression was measured using western blotting in SV-HUC-1 cells following TGF- $\beta 1$ stimulation. ${ }^{* *} \mathrm{P}<0.01$ vs. control. (B) shRNA-NC and shRNA-AQP-1/2 were used to transfect SV-HUC-1 cells and then AQP2 protein expression was detected by western blotting. ${ }^{* * *} \mathrm{P}<0.01$ and ${ }^{* * * *} \mathrm{P}<0.001$ vs. control. (C) SV-HUC-1 cells were treated with TGF- $\beta 1$ and transfected with pcDNA-FOXO1 alone or co-transfected with pcDNA-FOXO1 and shRNA-NC/shRNA-AQP2. Subsequently, cell viability was measured using Cell Counting Kit-8 assay. (D and E) Cell migration was measured using wound healing assay. ( $\mathrm{F}$ and $\mathrm{G}$ ) E-cadherin, $\mathrm{N}$-cadherin, cytokeratin, $\mathrm{FN}$ and $\alpha$-SMA protein expression levels were detected and analyzed using western blotting. ${ }^{*} \mathrm{P}<0.05$ and ${ }^{* * *} \mathrm{P}<0.001$ vs. control; ${ }^{\#} \mathrm{P}<0.05,{ }^{\# \#} \mathrm{P}<0.01$ and ${ }^{\# \#} \mathrm{P}<0.001$ vs. TGF- $\beta 1+\mathrm{Oe}-\mathrm{NC} ;{ }^{\$} \mathrm{P}<0.05,{ }^{\$ \$} \mathrm{P}<0.01$ and ${ }^{\$ \$ \$} \mathrm{P}<0.001$ vs. TGF- $\beta 1+O e-F O X O 1+$ shRNA-NC FOXO1, forkhead box protein O1; AQP2, aquaporin 2; shRNA, short hairpin RNA; NC, negative control; FN, fibronectin; $\alpha$-SMA, $\alpha$-smooth muscle actin.

to regulate multiple cellular processes, including cell proliferation, migration and apoptosis $(25,26)$. In the present study, only one binding site for the transcription factor FOXO1 was identified on the AQP2 promoter, which was verified using luciferase reporter and ChIP assays, suggesting that FOXO1 can interact with the DNA sequence in the promoter regions of $\mathrm{AQP} 2$ to contribute to the upregulation of AQP2. Subsequent experiments further revealed that FOXO1 overexpression 
upregulated AQP2 expression, which participated in the regulation of bladder fibrosis.

In conclusion, the results of the present study demonstrated that the expression of AQP2 was downregulated in TGF- $\beta 1$-induced bladder fibrosis. FOXO1-induced upregulation of AQP2 expression was found to protect against TGF- $\beta 1$-induced fibrosis by reducing cell viability, migration and EMT. These results may uncover a novel regulatory mechanism underlying bladder fibrosis and indicate a novel biomarker target.

\section{Acknowledgements}

Not applicable.

\section{Funding}

No funding was received.

\section{Availability of data and materials}

The datasets used and/or analyzed during the current study are available from the corresponding author on reasonable request.

\section{Authors' contributions}

ZL conceived and designed the study. YQ, ZX and ZG performed the experiments, and performed data mining, acquisition and analysis. ZL wrote and approved the final manuscript. ZL and YQ confirmed the authenticity of all the raw data. All the authors have read and approved the final manuscript.

\section{Ethics approval and consent to participate}

Not applicable.

\section{Patient consent for publication}

Not applicable.

\section{Competing interests}

The authors declare that they have no competing interests.

\section{References}

1. D'Silva KA, Dahm P and Wong CL: Does this man with lower urinary tract symptoms have bladder outlet obstruction? The rational clinical examination: A systematic review. JAMA 312: 535-542, 2014

2. Rademakers KL, van Koeveringe GA and Oelke M: Detrusor underactivity in men with lower urinary tract symptoms/benign prostatic obstruction: Characterization and potential impact on indications for surgical treatment of the prostate. Curr Opin Urol 26: 3-10, 2016.

3. Niemczyk G, Czarzasta K, Radziszewski P, Wlodarski P and Cudnoch-Jedrzejewska A: Pathophysiological effect of bladder outlet obstruction on the urothelium. Ultrastruct Pathol 42 : 317-322, 2018

4. Wiafe B, Adesida AB, Churchill T, Kadam R, Carleton J and Metcalfe PD: Mesenchymal stem cell therapy inhibited inflammatory and profibrotic pathways induced by partial bladder outlet obstruction and prevented high-pressure urine storage. J Pediat Urol 15: 254.e1-254.e10, 2019.
5. Metcalfe PD, Wang J, Jiao H, Huang Y, Hori K, Moore RB and Tredget EE: Bladder outlet obstruction: Progression from inflammation to fibrosis. BJU Int 106: 1686-1694, 2010.

6. Islam SS, Mokhtari RB, El Hout Y, Azadi MA, Alauddin M, Yeger $\mathrm{H}$ and Farhat WA: TGF- $\beta 1$ induces EMT reprogramming of porcine bladder urothelial cells into collagen producing fibroblasts-like cells in a Smad2/Smad3-dependent manner. J Cell Commun Signal 8: 39-58, 2014.

7. Jiang X, Chen Y, Zhu H, Wang B, Qu P, Chen R and Sun X: Sodium tanshinone IIA sulfonate ameliorates bladder fibrosis in a rat model of partial bladder outlet obstruction by inhibiting the TGF- $\beta$ /Smad pathway activation. PLoS One 10: e0129655, 2015.

8. Chen Y, Ma Y, He Y, Xing D, Liu E, Yang X, Zhu W, Wang Q and Wen JG: The TGF- $\beta 1$ pathway is early involved in neurogenic bladder fibrosis of juvenile rats. Pediatr Res: Jan 19, 2021 (Epub ahead of print).

9. Agre P and Kozono D: Aquaporin water channels: Molecular mechanisms for human diseases. FEBS Lett 555: 72-78, 2003.

10. Niu D, Bai Y, Yao Q, Zhou L, Huang X and Zhao C: AQP2 as a diagnostic immunohistochemical marker for pheochromocytoma and/or paraganglioma. Gland Surg 9: 200-208, 2020.

11. Kim SO, Choi D, Song SH, Ahn KY, Kwon D, Park K and Ryu SB: Effect of detrusor overactivity on the expression of aquaporins and nitric oxide synthase in rat urinary bladder following bladder outlet obstruction. Can Urol Assoc J 7: E268-E274, 2013.

12. Wang Y, Zhou Y and Graves DT: FOXO transcription factors: Their clinical significance and regulation. Biomed Res Int 2014: 925350, 2014

13. Xing YQ, Li A, Yang Y, Li XX, Zhang LN and Guo HC: The regulation of FOXO1 and its role in disease progression. Life Sci 193: 124-131, 2018.

14. Ide H, Mizushima T, Jiang G, Goto T, Nagata Y, Teramoto Y, Inoue S, Li Y, Kashiwagi E, Baras AS, et al: FOXO1 as a tumor suppressor inactivated via AR/ER $\beta$ signals in urothelial cells. Endocr Relat Cancer 27: 231-244, 2020.

15. Xin Z, Ma Z, Hu W, Jiang S, Yang Z, Li T, Chen F, Jia G and Yang Y: FOXO1/3: Potential suppressors of fibrosis. Ageing Res Rev 41: 42-52, 2018

16. Livak KJ and Schmittgen TD: Analysis of relative gene expression data using real-time quantitative PCR and the 2(-Delta Delta C(T)) method. Methods 25: 402-408, 2001.

17. Polyak K and Weinberg RA: Transitions between epithelial and mesenchymal states: Acquisition of malignant and stem cell traits. Nat Rev Cancer 9: 265-273, 2009.

18. Wang J, Chen Y, Gu D, Zhang G, Chen J, Zhao J and Wu P: Ketamine-induced bladder fibrosis involves epithelial-to-mesenchymal transition mediated by transforming growth factor- $\beta 1$. Am J Physiol Renal Physiol 313: F961-F972, 2017.

19. Lamouille S, Xu J and Derynck R: Molecular mechanisms of epithelial-mesenchymal transition. Nat Rev Mol Cell Biol 15: 178-196, 2014.

20. Qian W, Cai X, Qian Q, Zhang W and Wang D: Astragaloside IV modulates TGF- $\beta 1$-dependent epithelial-mesenchymal transition in bleomycin-induced pulmonary fibrosis. J Cell Mol Med 22: 4354-4365, 2018.

21. Iwano M: EMT and TGF-beta in renal fibrosis. Front Biosci (Schol Ed) 2: 229-238, 2010.

22. Shrestha N, Chand L, Han MK, Lee SO, Kim CY and Jeong YJ: Glutamine inhibits $\mathrm{CCl} 4$ induced liver fibrosis in mice and TGF- $\beta 1$ mediated epithelial-mesenchymal transition in mouse hepatocytes. Food Chem Toxicol 93: 129-137, 2016.

23. Lin S, Lian D, Liu W, Haig A, Lobb I, Hijazi A, Razvi H, Burton J, Whiteman $M$ and Sener A: Daily therapy with a slow-releasing $\mathrm{H}_{2} \mathrm{~S}$ donor GYY4137 enables early functional recovery and ameliorates renal injury associated with urinary obstruction. Nitric Oxide 76: 16-28, 2018.

24. Cardozo CP: Identification of transcription factor-binding sites in the mouse FOXO1 promoter. Methods Mol Biol 1890: 29-40, 2019.

25. Huang F, Wang Q, Guo F, Zhao Y, Ji L, An T, Song Y, Liu Y, He Y and Qin G: FoxO1-mediated inhibition of STAT1 alleviates tubulointerstitial fibrosis and tubule apoptosis in diabetic kidney disease. EBioMedicine 48: 491-504, 2019.

26. Yang L, Yang F, Zhao H, Wang M and Zhang Y: Circular RNA circCHFR facilitates the proliferation and migration of vascular smooth muscle via miR-370/FOXO1/cyclin D1 pathway. Mol Ther Nucleic Acids 16: 434-441, 2019.

This work is licensed under a Creative Commons Attribution-NonCommercial-NoDerivatives 4.0 International (CC BY-NC-ND 4.0) License. 